\title{
Use of Platelet-rich Plasma in the Treatment of Desmitis of Distal Interphalangeal Joint Collateral Ligament in a Horse
}

\author{
Geórgia Camargo Góss', Gabriela Döwich', Ingrid Rios Lima Machado², Fabricio Desconsi Mozzaquatro', \\ Ana Paula Rodrigues', Ana Luísa Moreira Carneiro', Carla Teixeira Leite³ \& Claudia Acosta Duarte'
}

\begin{abstract}
Background: Platelet-rich plasma (PRP) has been increasingly used to hasten healing of tendonitis and desmitis, allowing horse athletes to go back to practicing sports in a shorter period of time. However, its use has rarely been reported in the treatment of desmitis of the collateral ligaments of the distal interphalangeal joint (CL-DIP), which is based on the use of horseshoes and rest for at least six months. This paper describes the use of PRP as an aid in the treatment of CL-DIP in a horse athlete.

Case: A male Criollo horse, $450 \mathrm{~kg}$, about 15-year-old, used for paleteada. The animal had a month-long history of lameness, and a visual inspection revealed an increase in volume in the lateral dorsal region of the limb proximal to the coronary band of the hoof. A specific examination of the locomotive system showed Grade III, Category 5 claudication, which became evident the beginning of the exercise and ceased after a short period. A radiographic evaluation of the distal phalanx revealed no clinically relevant changes. Therefore, and in view of the location of the increased volume, an ultrasound scan was performed, which showed an image compatible with an extensive hematoma in the region underlying the perioplic corium, while the CL-DIP presented swelling with large areas of hypoechogenicity. Considering the findings of the image, the physical examination and the history of the horse, desmitis of the collateral ligaments of the distal interphalangeal joint was diagnosed. The treatment plan consisted of rest, application of a therapeutic horseshoe, and infiltration of the lesion with platelet-rich plasma (PRP). First, however, warm compresses were applied to reduce the swelling. The PRP was obtained by collecting whole blood from the external jugular vein, subjecting it to double centrifugation, and separating the supernatant blood plasma. The end product was applied to the lesion by ultrasound-guided infiltration. The horseshoe was a model with extended side bar, i.e., an egg bar horseshoe. After 15 days of PRP infiltration, another ultrasound scan was performed, which showed improved echogenicity of the ligament. The animal was discharged, and rest and the use of the horseshoe for 6 months were recommended. However, the owner expressed interest in having his horse participate in a high level team roping event, which was slated to take place two months hence. In view of this plan, rest was recommended for a further 15 days, followed by a gradual return to the exercise until the moment of the competition, which should be interrupted if the animal displayed any sign of lameness. The animal performed well in the competition, showing no loss of performance due to locomotor changes.

Discussion: Locomotor disorders in the digit region are common in Creole horses, and one of the affected structures in this area is the CL-DIP. Desmitis of this ligament is characterized by acute claudication in animals without radiographic abnormalities, and can be diagnosed based on ultrasound scans. The main cause of this disorder stems from biomechanical alterations, and therefore the main treatment is the use of a therapeutic bar horseshoe on the affected side. The prognosis varies between favorable and unfavorable, and the healing time lasts on average 6 months. In view of this lengthy period of time, one of the alternatives to hasten healing is to use PRP, which is commonly used in tendonitis of the flexor tendon and suspensory ligament desmitis. Nevertheless, this technique provides variable outcomes and its application to CL-DIP remains insufficiently described. In the case reported here, its use in association with conventional treatment was effective, enabling the horse to participate in competitive events without loss of performance due to claudication.
\end{abstract}

Keywords: healing, equine, athletic prognosis, regenerative therapy.

DOI: $10.22456 / 1679-9216.95766$ 


\section{INTRODUCTION}

Therapeutic alternatives have focused on the recovery of horses with ligament and tendon injuries to enable their rapid return to equine sports. Platelet-rich plasma (PRP) is a product of whole blood centrifugation and platelet separation that may hasten the healing of such injuries [15]. This biotechnique has been widely used in disorders of the locomotor system [3], mainly in the treatment of flexor tendon tendonitis [22] and suspensory ligament desmitis [19]. However, the outcomes of this technique are highly variable and its use in the treatment of desmitis of the collateral ligaments of the distal interphalangeal joint remains insufficiently described [20]. This condition, which is caused by biomechanical changes, is treated by means of therapeutic farriery [21] and resting for at least six months $[7,10]$. The prognosis varies between favorable to unfavorable, depending on the therapeutic efficacy, based on the level of exercise achieved after this period [10].

Thus, the purpose of this study was to examine the use of PRP as an aid in the treatment of desmitis of the collateral ligaments of the distal interphalangeal joint in a sporting horse.

\section{CASE}

This case involved a male Criollo horse, about 15-year-old, weighing $450 \mathrm{~kg}$, which was used for paleteada. His owner reported that the horse had presented lameness whenever he was taken out of his stall, which disappeared after a few minutes of exercise. The owner also stated that the animal had not previously shown signs of lameness.

A visual inspection revealed an increase in volume in the lateral dorsal region of the coronary band of the left forelimb (Figure 1A), but a physical examination indicated no clinical changes. A specific examination of the locomotor system revealed that the horse kept its affected limb on the ground but showed Grade III, Category 5 claudication when walking in a straight line, which became evident at the beginning of the exercise and ceased shortly thereafter. The horse showed no pain response to the hoof tester, but exhibited mild response to the distal interphalangeal joint (DIP) flexion test and pain response to digital pressure applied to the swollen area of the coronary band.

No changes were detected in the hematology tests. Imaging testing was performed using radiography and ultrasonography. The former, which involved a radiographic evaluation of the distal phalanx by means of the lateral medial, dorso-plantar and dorso-proximal palmaro-distal oblique projections at a $65^{\circ}$ angle, revealed no clinically relevant changes (Figure 1B). The ultrasound examination involved an evaluation of the swollen region, using the technique described by Denoix et al. [5]. The resulting image was compatible with hematoma in the region subjacent to the perioplic corium, making it difficult to examine the lateral collateral ligament of the DIP, which was swollen and had large areas of hypoechogenicity (Figure 1C).

The animal's history and clinical signs, allied to the findings of the complementary exams, especially the ultrasound, led to a diagnosis of desmitis of the lateral collateral ligaments of the DIP joint associated with hematoma. A therapeutic plan was therefore devised involving rest, intralesional PRP treatment, and corrective shoeing. However, the swelling prevented infiltration of the CL-DIP joint, so warm compresses were initially applied on the swollen area for 20 days until the swelling disappeared, allowing for the application of PRP.

The aforementioned therapy consisted of drawing $25 \mathrm{~mL}$ of blood from the external jugular vein, after antisepsis. The blood was stored in sodium citrate tubes, in which the initial platelet count was performed, showing 200,000 g/dL. The blood was allowed to rest for $5 \mathrm{~min}$, after which the tubes were centrifuged (Novatecnica NT $\left.812^{\circledR}\right)^{1}$ for $10 \mathrm{~min}$ at $120 \mathrm{~g}$. The centrifuged blood was allowed to rest for ten min, after which about $2 / 3$ of the supernatant was discarded. The remaining plasma was transferred to a tube without anticoagulant, and pipetting was done close to the phlogistic layer to avoid drawing up leukocytes and red blood cells as much as possible. This new tube was centrifuged for $10 \mathrm{~min}$ at $240 \mathrm{~g}$ and the upper half of the plasma was discarded. The remaining centrifuged material thus amounted to $2.5 \mathrm{~mL}$, with a final platelet count of 1,600,000 g/dL.

After obtaining the PRP, it was applied to the lesion, guided by ultrasound. To this end, the lateral dorsal region adjacent to the coronary band was shaved. The animal was restrained in a horse stock and was sedated with $0.01 \mathrm{mg} / \mathrm{kg}$ of detomidine hydrochloride $\left(\text { Eqdomin }^{\circledR}\right)^{2}$. After antisepsis, a linear ultrasound transducer was coated with sterile material, placed on the shaved area, and PRP was applied to the lesion by 
ultrasound-guided infiltration. Three days later, corrective farriery was applied using an egg bar shoe (Figure 2A). After 15 days, a new ultrasound scan showed improvement of the ligament fibers, indicated by a decrease in hypoechoic areas, while the swelling was visibly reduced and more homogeneous (Figure 2B). In addition, the volume of the coronary band in the area of the ligament had returned to normal (Figure 2C).

The horse was discharged after 35 days, and rest and the use of the corrective horseshoe for 6 months were recommended. However, the owner expressed interest in having his horse participate in a high level team roping event, which was slated to take place two months later. In view of this plan, rest was recommended for a further 15 days, followed by moderate training for a fortnight, and then gradually increase the level of physical exertion over the 30 days prior to the competition, provided the animal showed no sign of lameness. The horse performed well in the competition, showing no loss of performance due to locomotor changes.

\section{DISCUSSION}

Disorders of the digits are common in Criollo sports horses, and about $68.8 \%$ of cases of claudication in these animals occur in this region, many of which are radiographically diagnosed [1]. In this region, degenerative distal interphalangeal joint (DIP) disease and alar cartilage fracture are frequently diagnosed and require radiographic examination [2], which justifies the choice of this imaging exam as the first option in this particular case.

The absence of clinically significant radiographic alterations suggested soft tissue damage in the horse's hoof, and in this case, desmitis of the collateral ligaments of the DIP joint, which is a common condition

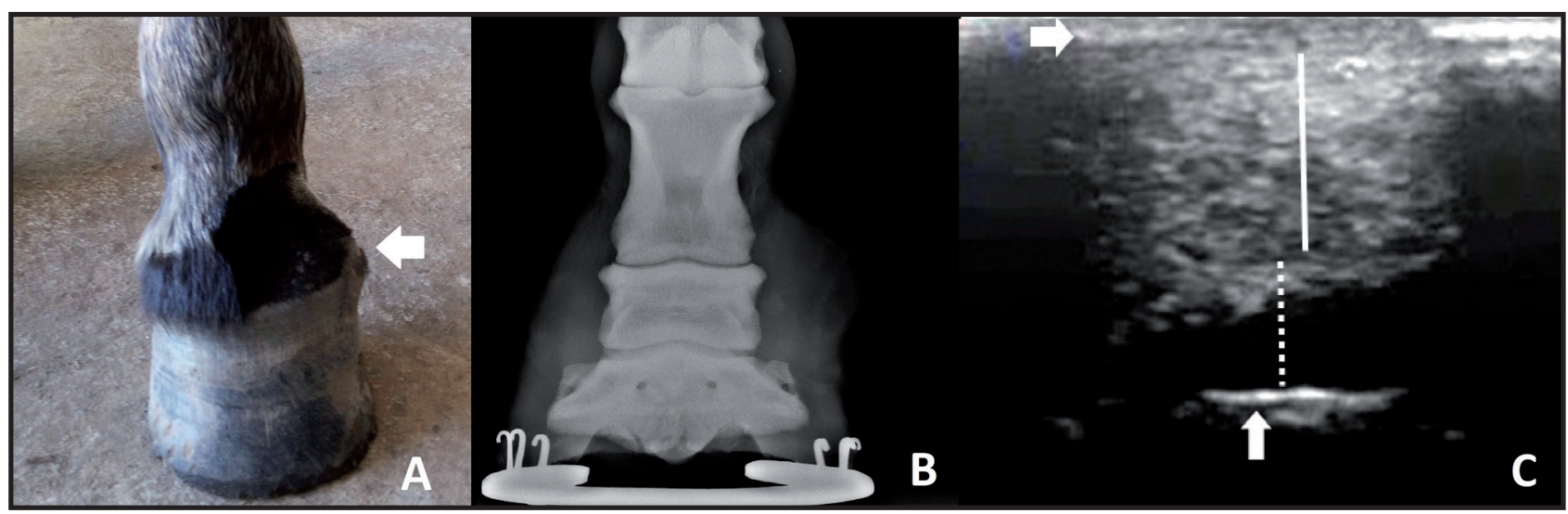

Figure 1. A- Volume increase in the dorsolateral region of the coronary band of the left thoracic limb (white arrow). B- Image of the dorsoplantar region of the hoof; note the absence of radiographic alterations. C- Ultrasound image of the dorsolateral region of the coronary band, using the distal concavity of the intermediate phalanx (longitudinal white arrow) as reference to locate the CL-DIP joint (white dotted line), which shows an extensive area of hypoechogenicity, undefined boundaries and unviewable ligament fibers. Note the area of moderate heterogeneous echogenicity subjacent to the perioplic corium (horizontal white arrow), suggestive of hematoma (white line).

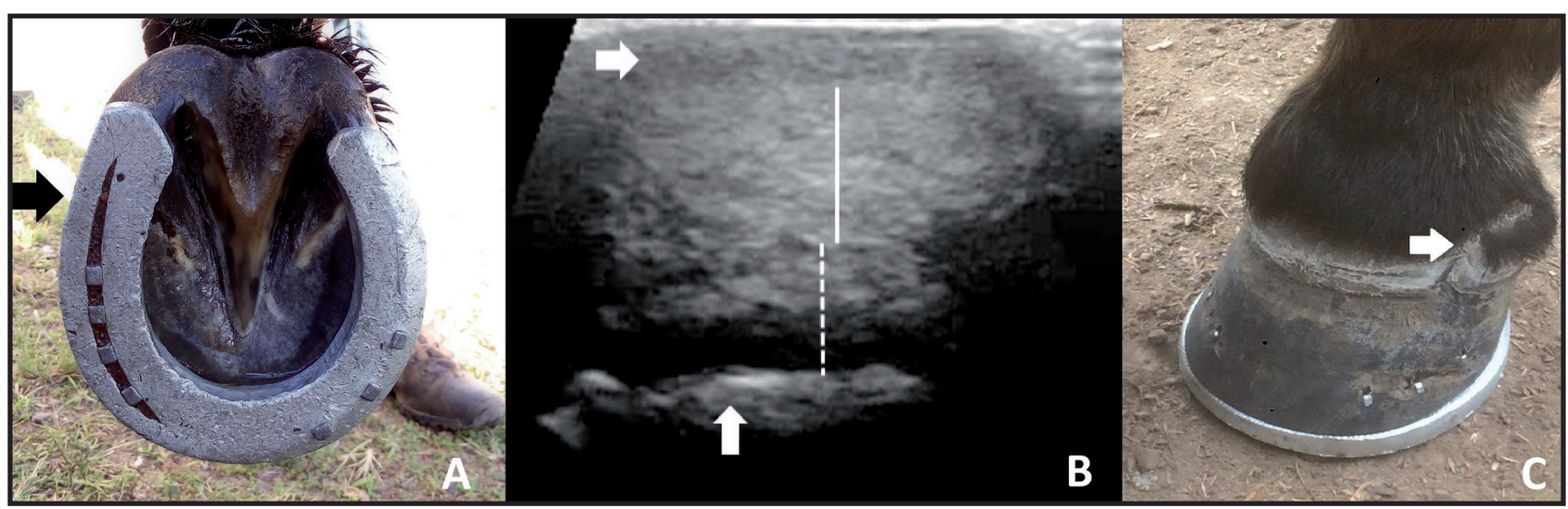

Figure 2. A- Aluminum egg bar horseshoe used on the horse with desmitis of the CL-DIP joint (black arrow). B- Cross-section of an ultrasound image of the CL-DIP joint (dotted white line) 15 days after PRP infiltration, using as reference the intermediate phalanx (longitudinal white arrow). C- Underlying the perioplic corium (horizontal white arrow) is an area suggestive of a moderately echogenic hematoma (white line), which is more homogeneous and less thick than the image taken prior to PRP infiltration. Note the larger more homogeneous area of echogenicity with clearer borders in the region of the CL-DIP joint. 
in animals with lameness originating from this region, without radiographic signs [8]. In these cases, ultrasound scanning is recommended [6]. In addition to this finding, the clinical signs corroborated the presumptive diagnosis, indicating the presence of Grade III, Category 5 claudication without painful response to the hoof tester [17], evidence of claudication after flexion of the distal interphalangeal joint [10], and swelling in the lateral dorsal region of the coronary band [8].

In view of the clinical signs, ultrasound imaging was chosen as the diagnostic method. This test has limitations for the diagnosis of desmitis of the CL-DIP joint, because only the proximal portion of the ligament can be visualized [5]. Nevertheless, it has proved to be effective for the diagnosis of this case and can be used as the first choice, or in situations where magnetic resonance is not an option. The ultrasound images showed areas of hypoechogenicity and edema, with consequently increased volume, which are the main changes observed when in this type of condition [6]. The clinical signs and ultrasound images of the horse of this case report explain the diagnosis of desmitis of the collateral ligaments of the distal interphalangeal joint.

The treatment consisted of rest, use of an egg bar horseshoe, and PRP infiltration. However, the hematoma had to be treated first, using warm compresses applied to the injured region, which helped to increase local blood flow and allowed the swelling to subside [13].

The egg bar horseshoe was chose because this model helps diminish the biomechanical strain to which sports animals are subjected, since this corrective horseshoe prevents stretching of the affected ligament at moments when the hoof is not positioned flat on the ground [21]. This horseshoe was effective in this case, enabling the horse to engage in team roping while wearing the shoe.

The other chosen therapy was platelet-rich plasma (PRP) infiltration, whose good results were attributed mainly to the final platelet concentration achieved. The PRP preparation method followed a previously described technique [4], which proved to be suitable. As expected, the final volume of PRP corresponded to $10 \%$ of the whole blood $[14,18]$. In this case, the final platelet concentration was about 8 times higher than the initial concentration, and although the final concentration is expected to be 3-5 times higher [10], the concentration obtained in this case was ideal and was also used successfully in another study [20]. In addition, the total infiltration of $2.5 \mathrm{~mL}$ of PRP has been previously described as the intralesional volume applied that achieved good results [12] and proved to be suitable for this case. Ultrasound-guided infiltration allowed the PRP injection to be administered correctly in the lesion, excluding the possibility of incorrect needle position, as reported by Lewis et al. [11]

Since the recovery time was shorter than that recorded in other cases of desmitis of the CL-DIP joint treated with corrective farriery [7], with cast immobilization [16], or in cases where PRP was used in combination with shoeing [20], it can be inferred that the use of PRP was an important therapeutic element in this case.

Less than 6 months sufficed for the horse in question to participate in competitive events again, demonstrating the effectiveness of the treatment and underscoring the fact that the proper early therapeutic intervention helped the prognosis of this case, preventing the early retirement of the horse. Moreover, it should be noted that the owner cooperated throughout the process, following the veterinary advice after the horse was discharged, enabling it to participate and perform well in the competitive event.

Thus, correcting the biomechanical change by means of a therapeutic horseshoe was essential. However, the use of PRP infiltration hastened the animal's recovery, improving the its prognosis and helping it return to its sports performance in a short period of time. It was concluded that platelet-rich plasma infiltration is a good alternative to assist in the treatment of desmitis of the CL-DIP joint.

\section{MANUFACTURERS}

${ }^{1}$ Novatecnica Ltda. Piracicaba, SP, Brazil.

${ }^{2}$ Ourofino Saúde Animal Ltda. Cravinhos, SP, Brazil.

Declaration of interest. The authors report no conflicts of interest. The authors alone are responsible for the content and writing of this paper. 


\section{REFERENCES}

1 Abreu H.C., De La Corte F.D., Brass K.E., Pompermayer E., Da Luz T.R.R. \& De Gasperi D. 2011. Lameness in athletic Criollo horses. Ciência Rural. 41(12): 2114-2119.

2 Baxter G.M. \& Stashak T.S. 2011. The foot. In: Baxter G.M. (Ed). Adams and Stashak: Lameness in Horse. 6th edn. Hoboken: Wiley-Blackwell, pp.505-623.

3 Brossi P.M., Moreira J.J., Machado T.S.L. \& Baccarin Y.A. 2015. Platelet-rich plasma in orthopedic therapy: a comparative systematic review of clinical and experimental data in equine and human musculoskeletal lesions. BMC Veterinary Research. 11(98): 1-17.

4 Carneiro A.L.M., Duarte C.A., Döwich G., Góss G.C., Lagreca L.F.J. \& Bataglin C.F. 2017. Alternativa de protocolo para obtenção de plasma rico em plaquetas (PRP) em equinos. In: Resumos do XIX Salão Internacional de Ensino, Pesquisa e Extensão (Santana do Livramento, Brasil). pp.1-3.

5 Denoix J.M., Bertoni L., Heitzmann A.G., Werpy N. \& Audigié F. 2011. Ultrasonographic examination of the collateral ligaments of the distal interphalangeal joint in horses: Part A: Technique and normal images. Equine Veterinary Education. 23(11): 574-580.

6 Denoix J.M., Bertoni L., Heitzmann A.G., Werpy N. \& Audigié F. 2011. Ultrasonographic examination of the collateral ligaments of the distal interphalangeal joint in horses. Part B: Abnormal findings and lesions. Equine Veterinary Education. 23(12): 616-625.

7 Dyson S.R., Murray R. \& Schramme M.C. 2005. Lameness associated with foot pain: results of magnetic resonance imaging in 199 horses (January 2001-December 2003) and response to treatment. Equine Veterinary Journal. 37(2): 113-121.

8 Dyson S.J., Murray R., Schramme M. \& Branch M. 2004. Collateral desmitis of the distal interphalangeal joint in 18 horses (2001-2002). Equine Veterinary Journal. 36(2): 160-166.

9 Gonshor A. 2002. Technique for producing platelet-rich plasma and platelet concentrate: background and process. The International Journal of Periodontics and Restorative Dentistry. 22(6): 547-557.

10 Gutierrez-Nibeyro S.D., White N.A., Werpy N.M., Tyrrell L., Allen K.A., Sullins K.E. \& Mitchell R.D. 2009. Magnetic resonance imaging findings of desmopathy of the collateral ligaments of the equine distal interphalangeal joint. Veterinary Radiology \& Ultrasound. 50(1): 21-31.

11 Lewis D., Scott M., Fischer C.D., Bond S.L. \& Léguillette R. 2016. Feasibility for ultrasound-guided injection of the collateral ligaments of the distal interphalangeal joint in horses. Veterinary Radiology and Ultrasound. 57(3): 299-305.

12 Maia L., Souza M.V., Alves G.E.S., Júnior J.I.R., De Oliveira A.C., Zandim B.M. \& Da Silva Y.F.R.S. 2009. Platelet-rich plasma in the treatment of induced tendinitis in equines: Ultrasonographic evaluation. Pesquisa Veterinária Brasileira. 29(3): 241-245.

13 Mehra P., Clancy C. \& Wu J. 2000. Formation of a facial hematoma during endodontic therapy. Journal of American Dentistry Association. 131(1): 67-71.

14 Pereira R.C.F., Zacarias G.V.F., Cantarelli C., Corrêa M.M.B., Da Silva G.B., Barbosa A.L.T., Brass K.E. \& De La Corte F.D. 2013. Evaluation of seven platelet-rich plasma processing protocols in the equine species. Ciência Rural. 43(6): 1112-1127.

15 Rindermann G., Cislakova M. \& Arndt G. 2010. Autologous conditioned plasma as therapy of tendon and ligament lesions in seven horses. Journal of Veterinary Sciences. 2(1): 173-175.

16 Trope G.D. \& Whitton R.C. 2009. Medial collateral ligament desmitis of the distal interphalangeal joint in the hindlimb of a horse: treatment with cast immobilisation. Australian Veterinary Journal. 87(12): 499-502.

17 Turner T.A. \& Sage A.M. 2002. Desmitis of the Distal Interphalangeal Collateral Ligaments: 22 Cases. In: Proceedings of the 48th American Association of Equine Practitioners Annual Convention. (Orlando, USA). pp.343-346.

18 Vendruscolo C.P., Carvalho A.M., Moraes L.F., Maia L., Queiroz D.L., Watanabe M.J., Yamada A.L.M. \& Alves A.L.G. 2012. Evaluating the effectiveness of different protocols for preparation of platelet rich plasma for use in equine medicine. Pesquisa Veterinária Brasileira. 32(2): 106-110.

19 Waselau M., Sutter W.W., Genovese R.L. \& Bertone A.L. 2008. Intralesional injection of platelet-rich plasma followed by controlled exercise for treatment of midbody suspensory ligament desmitis in Standardbred racehorses. Journal of the American Veterinary Medical Association. 10(232): 1-6. 
20 White N.A. \& Barret J.G. 2016. Magnetic resonance imaging-guided Treatment of equine Distal interphalangeal Joint collateral ligaments: 2009-2014. Frontiers of Veterinary Sciences. 3(1): 1-6.

21 Zubrod C.J., Farnsworth K.D., Tucker R.L. \& Ragle C.A. 2005. Injury of the collateral ligaments of the distal interphalangeal joint diagnosed by magnetic resonance. Veterinary Radiology \& Ultrasound. 46(1): 11-16.

22 Zuffova K., Krisova S. \& Zert Z. 2013. Platelet rich plasma treatment of superficial digital flexor tendon lesions in racing Thoroughbreds. Veterinární Medicina. 58(4): 230-239. 